\title{
Application of Surface Plasmon Resonance (SPR) for the Detection of Single Viruses and Single Biological Nano-objects
}

\section{Shpacovitch Victoria*}

ISAS, Leibniz Institute for Analytical Sciences, Dortmund, Germany

Real-time and label-free optical biosensors based on the principles of surface plasmon resonance (SPR) phenomena are widely used to study interactions between different biomolecules (nucleic acids, proteins, peptides). Despite many advantages of SPR-based instruments, they remained useless for detection of single nanosized biological objects such as viruses or virus-like particles. However, recent groundbreaking studies demonstrated suitability of modified SPR-based imaging techniques for the detection of single viruses and visualization of viral binding events.

\section{Editorial}

Surface plasmon resonance (SPR) based biosensor became commercially available from Biacore (GE Healthcare) in 1990 [1]. Today SPR-based biosensors are widely used and significantly contribute to the studies of interactions between different types of biomolecules (nucleic acids, receptors, peptides, proteins, antibodies, lipids) [2-4]. Moreover, it is worth to mention that SPR-based biosensors can help to investigate different aspects of viral binding to functionalized surfaces and are also useful as anti-viral drug discovery tools $[5,6]$.

SPR is a label-free, high sensitive optical method of analysis. It is worth to present certain explanations of basic principles of SPR-based biosensor instrument omitting relatively complex physical principles of the SPR phenomena (Figure 1). In the classical SPR approach, a biomolecule is immobilized onto a sensor surface (usually a noble metal is used as a sensor) and an interacting (binding) bio-particle becomes available from an analyzed probe. Interactions between bioparticles occur at sensor's interface in flow cell system under continues flow. The binding of immobilized and floating biomolecules leads to the accumulation of biomolecules onto the sensor's surface and, thus, results in increase of the refractive index near to the sensor surface. Changes of the refractive index lead to changes in surface plasmon waves (or surface plasmon polarations, SPP). Changes of refractive index may be measured in real time and thus, provide information about binding efficiency of biomolecules without any time-delay (Figure 1).

Among the advantages of the SPR method, one can mention its ability to perform real-time observation in the binding of label-free biomolecules. Real-time detection enables to determine the rates of association and dissociation during binding events. Unfortunately, the use of fluorescent, magnetic or radioactive labels may interfere with binding process, for example, by occluding a binding site. Under such circumstances label-free methods, like SPR, are the most desirable. Moreover, relatively simple modifications of biosensor surface allow to create different types of surface functionalization and, thus, to study interactions between different biomolecules with minimum changes of the experimental setup. The sensitivity of SPR makes it useful for the analysis of relatively small quantities of purified bioparticles [4].

However, there are also certain disadvantages in the use of SPR technique. For example, the use of SPR-based instruments for concentration measurements may be complicate and inconvenient. In addition, SPR method remained, up to recent achievements, useless for detection of single viral particles as well as other types of single nanoparticles.

The majority of viruses (excluding certain phages) represent a type of nano-sized biological objects. Despite viruses are small, their prompt detection and quantification remain extremely important for many aspects of human life including precise disease diagnostics, therapeutics, control of food and water supplies. There are several commonly used methods for detection, characterization and quantification of viruses. Among these methods are PCR or RT-PCR, REA, ELISA and other types of immunoassays. These methods are effective, but they are often time consuming and require specific sample preparation. Moreover, some of these methods are also limited by quantity of the analyzed material. However, in the ideal situation, SPR imaging (SPRi) technique could be a serious competitor of immunoassay-based and nucleic acidbased methods of viral detection. Ideal SPRi technique may offer highly sensitive, specific, reproducible, rapid and relatively cheap detection of different viruses in solutions and in air. In spite, lateral resolution in SPR was considered as a limiting factor influencing the minimum size (micrometers) of visualized objects [7-9], recent groundbreaking studies made possible the detection of single nano-objects using modified SPRi technique [10-12].

In the study performed in 2010, Zybin and colleagues used SPRbased microscopy to detect the binding of single nanoparticles to the functionalized gold sensor surface [10]. Images of polystyrol (80 $\mathrm{nm}$ diameter) nanoparticles were obtained using SPRi technique. It was possible to visualize the binding of any single nanoparticle onto the sensor surface. Moreover, the binding of HIV virus-like particles (around $100 \mathrm{~nm}$ diameter) to the antibody-coated sensor surface was also monitored [10]. In addition, suggested experimental set up made potentially possible the monitoring of binding events at a relatively big surface area of the sensor (it may reach $10 \mathrm{~mm}^{2}$ ). This feature ensures high concentration sensitivity of the described modified SPRi technique. The suitability of the suggested SPRi technique for concentration measurements of viruses or virus-like particles in solutions is discussed in the next study presented by the same working group [12].

Wang and colleagues also demonstrated the applicability of SPR imaging for the detection of single viruses [11]. In this study, influenza A H1N1 viral particles were used. Tracking the particle images over the

*Corresponding author: Victoria Shpacovitch, Department of Interface Analysis, ISAS, Leibniz Institute for Analytical Sciences, Bunsen-Kirchhoff str.11, Dortmund 44139, Germany, Tel: +49(0)231-1392230; E-mail: vshpacovi@googlemail.com

Received September 21, 2012; Accepted September 22, 2012; Published September 25, 2012

Citation: Victoria S (2012) Application of Surface Plasmon Resonance (SPR) for the Detection of Single Viruses and Single Biological Nano-objects. J Bacteriol Parasitol 3:e110. doi:10.4172/2155-9597.1000e110

Copyright: () 2012 Victoria S. This is an open-access article distributed under the terms of the Creative Commons Attribution License, which permits unrestricted use, distribution, and reproduction in any medium, provided the original author and source are credited. 
Citation: Victoria S (2012) Application of Surface Plasmon Resonance (SPR) for the Detection of Single Viruses and Single Biological Nano-objects. J Bacteriol Parasitol 3:e110. doi:10.4172/2155-9597.1000e110

Page 2 of 3

A

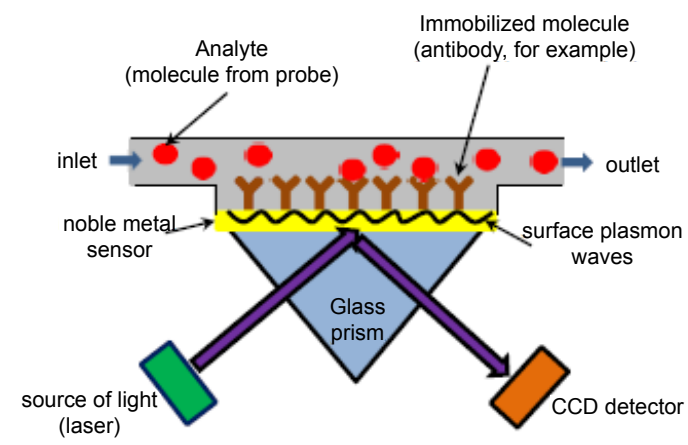

B

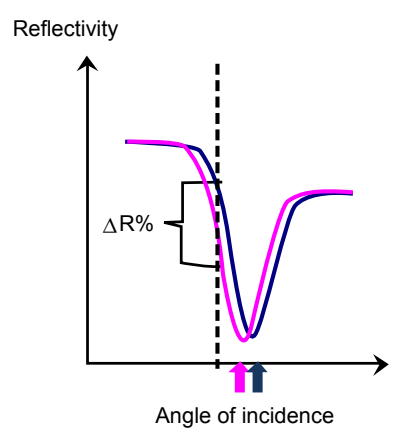

Figure 1: A scheme of a possible experimental set up for a surface plasmon resonance (SPR)-based instrument (using flow conditions). (A) A glass prism is coated with a thin layer of a noble metal (usually gold) to create a biosensor surface. Antibody or another type of biomolecule (named "ligand") is immobilized on the sensor surface (A). The metal layer possesses surface mobile electrons. At certain incidence angle the incoming beam of light disturbs these electrons causing changes in surface plasmon waves (A). Electrons "resonate" giving a name to SPR phenomena. This particular incidence angle is called SPR angle. At the SPR angle reflectivity drops to a minimum (B). However, the binding of antibody to free analyte onto sensor's surface (A) leads to accumulation of biomolecules onto surface and subsequently affects SPR conditions (B). Refractive index increases and a shift of SPR angle occurs (B). SPR-based methods offer two opportunities to measure the efficiency of binding to sensor's surface: 1) measuring the shift of SPR angle during binding of biomolecules; 2) when SPR angle remains fixed- measure changes of reflectivity $(\Delta \mathrm{R} \%)(\mathrm{B})$

time allowed the authors to demonstrate that virus-surface interactions depends on the type of used functionalized surface (bar gold, PEGcoated or anti-influenza A antibody coated sensor). Moreover, the authors characterized the intensity profiles of the signals received from a single viral particle. In addition, SPR imaging was applied for the determination of mass and size of investigated influenza A viral particles. The results of viral mass and size measurements performed in this study using SPRi technique were in good agreement with previously published data [11].

Altogether, the above mentioned studies clearly demonstrate the suitability of the modified and optimized SPRi technique for the detection of single viruses or virus-like particles in solutions. These proof-of-principle works are very important for further improvement of the sensitivity of SPR microscopy as well as for the development of special software for the data processing. Data processing in the described studies $[10,11]$ was performed with ImageJ or MatLab package. Unfortunately, this cannot be considered as an optimal solution for the SPR image processing. Finding of other algorithm (s) is desirable for the enhancement of the data analysis quality. However, proof of principle by itself is undoubtedly a significant achievement and serves as a basis for future applications of SPRi techniques in viral studies and other research dealing with biological nano-size particles. For example, described modified SPRi technique appears to be suitable for the discrimination between exosomes and viruslike particles (VLPs) in standard VLP preparations. This issue may be important for the characterization and quality control of VLP-based vaccine stocks. Exosomes (30-200 nm diameter) represent a sub-group of small membrane vesicles and together with so-called microvesicles (200-1000 nm diameter) belong to extracellular vesicles. Extracellular vesicles are released from most animal cells. Their role in intercellular communication as well as their ability to serve as potential disease biomarkers is currently intensively studied $[13,14]$. Application of SPRi method for the detection and characterization of extracellular vesicles looks as a very useful and attractive opportunity. Further, SPRi technique may be suitable for characterization of association and dissociation rates during the binding of drug-carrying nano-particles with different types of biomolecules (mucins, receptors, proteins). It is extremely important to notice that modified SPRi technique allows visualizing binding events of a single biological nano-particle and, thus, potentially may be useful for concentration measurements based on the signal counting [12].

In summary, modified SPRi method, which allows detection and visualization of single biological nano-particles, certainly provides new fascinating opportunities in such research areas as viral biology, biology of extracellular vesicles, characterization of novel disease biomarkers and development of drug-delivering nanoparticles.

\section{Acknowledgements}

This work of Dr. Victoria Shpacovitch is supported by German Ministry of Science and High Education (Deutsche Forschungsgemeinschaft) grant SFB 876 Project B2.

\section{References}

1. Owen V (1997) Real-time optical immunosensors - a commercial reality Biosens Bioelectron 12: 1-2.

2. Scarano S, Mascini M, Turner APF, Minunni M (2010) Surface plasmon resonance imaging for affinity-based biosensors. Biosens Bioelectron 25: 957 966.

3. Kodoyianni V (2011) Label-free analysis of biomolecular interactions using SPR imaging. BioTechniques 50: 32-40.

4. Homola J (2006) Surface plasmon resonance based sensors. Springer Series on Chemical Sensors and Biosensors (Springer-Verlag, Berlin-Heidelberg_ New-York).

5. Caygill RL, Blair GE, Millner PA (2010) A review on viral biosensors to detect human pathogens. Analyt Chim Acta 681: 8-15.

6. Rich RL, Myszka DG (2003) Spying on HIV with SPR. Trends Microbiol 11 124-133.

7. Rothenhauser B, Knoll W (1988) Surface plasmon microscopy. Nature 332 615-617.

8. Berger CEH, Kooyman RPH, Greve J (1994) Resolution in surface plasmon microscopy. Rev Sci Instrum 65: 2829-2836.

9. Berger CEH, Kooyman RPH, Greve J (1999) Surface plasmon propagation near an index step. Opt Comm 167: 183-189.

10. Zybin A, Kuritsyn YA, Gurevich EL, Temchura VV, Überla K, et al. (2010) Real-time detection of single immobilized nanoparticles by surface plasmon resonance imaging. Plasmonics 5: 31-35. 
Citation: Victoria S (2012) Application of Surface Plasmon Resonance (SPR) for the Detection of Single Viruses and Single Biological Nano-objects. J Bacteriol Parasitol 3:e110. doi:10.4172/2155-9597.1000e110

Page 3 of 3

11. Wang S, Shan X, Patel U, Huang X, Lu J, et al. (2010) Label-free imaging, detection, and mass measurement of single viruses by surface plasmon resonance. PNAS 107: 16028-16032.

12. Gurevich EL, Temchura VV, Überla K, Zybin A (2011) Analytical features of particle counting sensor based on plasmon assisted microscopy of nano objects. Sensors and Actuators 160: 1210-1215.
13. Cocucci E, Racchetti G, Meldolesi J (2008) Shedding microvesicles: artefacts no more. Trends Cell Biol 19: 43-51.

14. Piccin A, Murphy WG, Smith OP (2007) Circulating microparticles: pathophysiology and clinical implications. Blood Rev 21: 157-171. 